\title{
CHEMICAL OXYGEN-IODINE LASER BASED ON HIGH PRESSURE SINGLET GENERATOR
}

M.V. ZAGIDULLIN, V.D. NIKOLAEV, A.Y. KUROV, M.I. SVISTUN and N.E. YERASOV

Laser Laboratory, P.N. Lebedev Physical Institute AS USSR, Samara Branch,

Novo-Sadovaya St. 221, Samara 443011, USSR

Abstract. - The singlet oxygen generator based on the injection of jets of the base hydrogen peroxide solution into chlorine llow has been developed. The optimal parameters of the jet generator were found to achieve efficient chlorine utilization and high $O_{2} i^{1} \Delta$ yield up to the pressures of 30 torr. The chemical oxygentiodine laser performance without water vapor trap up to 30 tors of the generator pressure has been at tained.

\section{1. - Introduction.}

The chemical oxygen-iodine laser (COIL) is based on the resonant energy transfer between $O_{2}{ }^{1} \Delta$ and iodine atoms $[1]$. It usually uses the singlet oxygen generators (SOG) that create high $0_{2} i^{1} \Delta$ yield with high chlorine utilization at the low exiting pressures (several torrs) only. One has to place the water vapor trap between the SOO and the resonator body of the COIL. Increasing the overall oxygen pressure up to 30 torr and provinding the solution temperature is $-10^{\circ} \mathrm{C}$ allows to achieve desirable water vapor contents at the soo outlet. Moreover, some efficient schemes are possible for gas exhaustion at supersonic COIL operation. In this work the sog based on continuous injection of jets of the hydrogen peroxide solution into chlorine flow has been developed. This type sog operates up to 30 torr of the initial chlorine pressures with high $O_{2} t^{1} \Delta y i e l d$ and efficient chlorine utilization. The subsontc COIL operation has been achieved up to 30 torr of sog pressures. 


\section{2. -Experinental set-up.}

The design of the continuous jet SOG is very similar to the pulsed one described earlier [Z]. In the jet sog solution jets move in the direction opposite to that of the chlorine flow. The design of the jet sog permits to change the number of jets, gas and jets velocities, gas-liquid interaction length. The upper and 1 ower tank for the solution and the jet soo body are supported at $-10^{\circ} \mathrm{c}$. The partial pressures of $\mathrm{O}_{2}{ }^{1} \Delta, \mathrm{Cl}_{2}$ and $\mathrm{H}_{2} O$ are measured at the exit of the jet soo. After the jet soo outlet the oxygen pressure is released 15 times by means of gas constrictors and then the oxygen is introduced into the resonator body. The active length of the laser duct is $25 \mathrm{~cm}$, the height is $7 \mathrm{~mm}$. The iodine vapor is carried out from the radiatively heated iodine cell by the argon $f(0)$ and transversly injected into the oxygen flow.

\section{3. -Jet soG modelling.}

The jet sog modeliting is based on the surface reaction approximation 131. In this model the kinetics of chlorine absorbtion by solution is described by the gas-liquid mass tranfser coefficient $B(\mathrm{~cm} / \mathrm{s})$ depended on the $\mathrm{HO}_{2}{ }^{-}$ions concentration on the solution surface. The HO ${ }_{2}^{-}$concentration depletion on the jet surface is described by the diffusion equation with the boundary condition on the jet surface. Joint solution of the equations for $\mathrm{O}_{2}{ }^{1} \mathrm{~A}, \mathrm{Cl}_{2}$ in a gas phase and for the $\mathrm{HO}_{2}^{-}$along the jet $\mathrm{SOO}$ axis allows to calculate the $\mathrm{Cl}_{2}$ utilization, the $\mathrm{O}_{2}{ }^{1} \Delta$ yield at the exit of jet sog vs its initial parameters.

\section{4. -Jet 800 testing}

a) Measurements of the chl orine absorbtion rate.

The jets in the sOG body for this measurements were produced by the injector with 45 holes of $0,3 \mathrm{~mm}$ in diameter. The velocity of the $\mathrm{KOH}-\mathrm{H}_{2} \mathrm{O}_{2}-\mathrm{H}_{2} \mathrm{O}$ solution jets equaled to $7 \mathrm{~m} / \mathrm{s}$. The Ar-Cl $=9: 1$ mixture at a low pressure was flowing through the sog. The efficiency of chlorine absorbtion vs the gas-liquid contact time and the KOH concentration was measured. Assuming that the contact surface was equal to the surface of 45 smooth jets of $0,3 \mathrm{~mm}$ in diameter we deduced the mass-transfer rate $\beta=\beta_{0}\left[K O H 1^{0,0}\right.$, where $\beta_{0}=(146 \pm 60) \mathrm{cm} / \mathrm{s}$ $M^{0.5}$. Taking into account the theoretical formular for $\beta[3]$ we estimate the rate constant for the reaction

$K=410^{8} \mathrm{M}^{-1} \mathrm{~s}^{-1}$ in agreement with [4].

$$
\mathrm{Cl}_{2}+\mathrm{HO}_{2}^{-}-2 \mathrm{Cl}-+\mathrm{H}^{+}+\mathrm{O}_{2}{ }^{2} \Delta
$$


b) Performance of the jet $50 \mathrm{O}$ at high chlorine pressures.

The $O_{2} i^{1} \Delta$ yield and the residual $\mathrm{Cl}_{2}$ traces at the high initial chlorine pressures are represented in Fig 1 . In this case the gas relocity was $10 \mathrm{~m} / \mathrm{s}$, the jets veloolty $7 \mathrm{~m} / \mathrm{s}$, the length of gas-li quid contact $10 \mathrm{~cm}$. The measurements showed that increasing of the gas flow veloctty and decreasing of the KOH concentration result in increasing of $\mathrm{Cl}_{2}$ traces. The computer analysis of this and other results allowed us to reach the conolusion that the renovation of the jets surface by $\mathrm{HO}_{2}^{-}$lons is supported mainly by the molecular diffusion mechanism and the role of hydrodinamic processes are negligible. Decreastng of the jets veloeity results in increasing of $\mathrm{Cl}_{2}$ traces. At higher initial $\mathrm{Cl}_{2}$ pressures this effect is stronger. Injection of mixiure $\mathrm{Cl}_{2}: \mathrm{Ar}=1: 1$ into jet sOG allowed to increase the overall exiting pressure up to 60 torr without significant decrease of $O_{2} r^{1} \Delta$ yilld and increase of chlorine traces at the exit of the soo.

$\left.0_{2}{ }^{1} \Delta\right), \%$

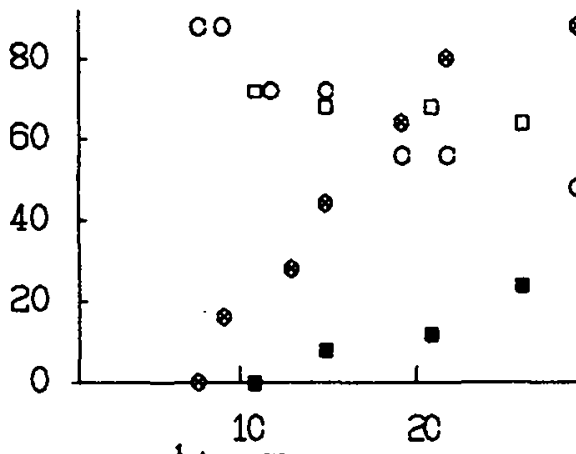

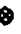

$\mathrm{Cl}_{2}, \%$

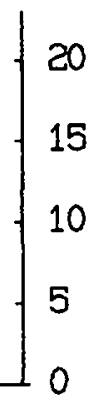

X
40 torr

pressure.

circle-injector 140 holes of $0,3 \mathrm{~mm} \mathrm{i.d.}$ square-inject or 280 hol es of $0,3 \mathrm{~mm} 1 . \mathrm{d}$. dark-Cl $\left.2^{\prime} 11 \mathrm{ght}-\mathrm{O}_{2}\right\}^{t} \Delta$

c) Wat or vapor pressure

The tests of the jet 500 showed that water vapor pressure at the exit was near its saturated value at $-10^{\circ} \mathrm{C}$. Thus the heat effect of the chlorination reaction is effectively compensated by the solution mass flow rate.

5. - Coll perforence with jet sog

The main goal of the COIL test with the jet soo was to demonstrate the possibility of its operation at high oxygen 
pressures at the exit of the jet soo. For this test pure chlorine was introduced into the jet soo body. Then the gas mixture from the jet soo was injected into resonator body through measuring cavity. The system of the gas constrletors provided the gas pressure relation $15: 3: 1$ at the exits of the sog, the measuring cavity and the resonat or cavity accordingly. The resonator cavity $80 \mathrm{~cm}$ in length consists of two spherical mirrors $\left(r=5 \mathrm{~m}, R_{1}=100 \%, R_{2}=99 \%\right.$. The molar flow rate of $\mathrm{I}_{2}$ was 30 mol/s for any oxygen pressure. The output COIL power vs exiting sog pressure is represented in Fig. 2 .

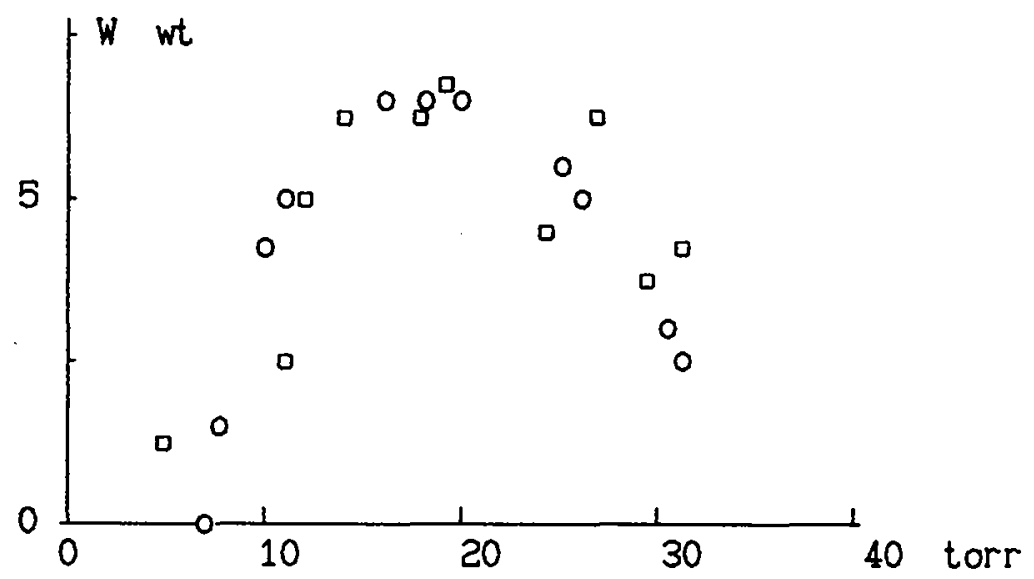

Fig.2. The output power vs exiting jet sog pressure O-jet injector 140 hol es of $0,3 \mathrm{~mm} 1 . \mathrm{d}$ a jet injector 280 holes of $0,3 \mathrm{~mm} 1 . \mathrm{d}$

The overall COIL efficiency is less than $5 \%$ in the best case. Nevertheless we beliteve that future improvement of the lodine mixing, the optimization of iodine injector -optical axis distance and resonat or coupling will allow us to increase the overall effleiency. 6. -Conclusions.

The jet sog is a very effective source of $0_{2} i^{2} \Delta$ with high energy density. The possibility of the coIL operation up to 30 torr of the exiting sOg pressures has been demonstrated. A maximum power 7 wt at 20 torr of extting soo pressure has been at tat ned. 1.W. E. McDermott,N. R. Pchel ki n,D. J. Benard,R. R. Bousek. Appl . Phys. Let ts.,

Z.N.F. Bal an, R.M. Gizatullin, M.V. Zagiduli in, A.Y.Kurov, V. D. Nikol aev, V.M.Pichkasov, M.I.Svistun. Krantovaya Electrontca, 16, 2197 (1989)

3. R. J. Ri chardson,J. D. Kel l ey,C. E. Wi swal I. J. Appl. Phys. 52, 1066(1981)

4. R. J. Ri chardson, P.A.G.Carr, F.E. Hovis, H.Y.Ageno, J.K. Hurst. J. Appl. Phys. 53,3272 (1982). 